\title{
Pyrimidine Dimer
}

National Cancer Institute

\section{Source}

National Cancer Institute. Pyrimidine Dimer. NCI Thesaurus. Code C790.

A pair of pyrimidine bases (thymine or cytosine in DNA or uracil in double-stranded RNA) within a nucleic acid covalently linked by a cyclobutane ring, the formation of which was induced by exposure to ultraviolet radiation. The presence of pyrimidine dimers inhibits DNA replication; improper repair of these lesions promotes DNA mutagenesis. 